\title{
Multipartite monogamy of the concurrence
}

\author{
Marcio F. Cornelio \\ Instituto de Física, Universidade Federal de Mato Grosso, Cuiabá - MT, 78068-900, Brazil
}

\begin{abstract}
Monogamy of entanglement is generally discussed using a bipartite entanglement measure as an upper bound. Here we discuss a new kind of monogamous relation where the upper bound is given by a multipartite measure of entanglement, the generalized concurrence. We show a new monogamous equality involving the multipartite concurrence, all the bipartite concurrences and the genuine tripartite entanglement for pure three qubits system. The result extends to mixed states in an inequality involving the generalized concurrence and all the bipartite concurrences. We provide a counter-example showing that the result cannot be extended for systems with more than three qubits.
\end{abstract}

One of the most fundamental properties of quantum correlations is that, differing from classical correlations, they are not shareable at will when distributed among many parties. A simple example is a pure maximally entangled state shared between Alice and Bob. This state cannot share any additional correlation (classical or quantum) with other parties. The composite system with a third party, say Carol, can only be a tensor product of the state of Alice and Bob with the state of Carol. This property has been called monogamy of entanglement and there is a vast literature about [1-6]. The concept of monogamy is important for many tasks in the quantum information theory, particularly, in the quantum cryptography [7]. In addition to the monogamy of entanglement, the concept of monogamy has also appeared when discussing the violation of the Bell's inequalities [8]. Also, it has been recently discussed in the context of quantum correlations [9] like the quantum discord [10]. Although, it has been shown that quantum correlation measures cannot satisfy the traditional monogamous equation [9], it has been shown it does satisfy a constrain with entanglement of formation [11, 12] called conservation law working in a similar spirit to monogamy [13]. In this work, we will be restricted to the discussion of the monogamy of entanglement. However, we shall see that, even in this case, one can find new relations that go beyond the traditional monogamous inequality (defined below in Eq. (3)). We find a new monogamous equality involving the multipartite generalized concurrence $[14,15]$ which extends to an inequality for mixed states. It seems to be the first time that monogamy is discussed using a multipartite measure of entanglement as the upper bound to other measures. In addition, we show that the result cannot be extend to systems with more than three qubits. We believe that the reason for this problem is that the generalized concurrence is not an additive measure.

The firsts to quantify this intuitive property of entanglement were Coffman, Kundu and Wootters [1] in 2000. They have shown that, for a pure three qubit state $\varphi_{a b c}$, the measure of entanglement called concurrence $[11,16]$ satisfies the following equality

$$
C_{a \mid b c}^{2}\left(\varphi_{a b c}\right)=C^{2}\left(\rho_{a b}\right)+C^{2}\left(\rho_{a c}\right)+\tau,
$$

where $C_{a \mid b c}$ stands for the concurrence between the subsystems $S_{a}$ and $S_{b c}$ and $\tau$ stands for the genuine tripartite entanglement. As the state $\varphi_{a b c}$ is pure, the squared concurrence $C_{a \mid b c}^{2}$ is just the linear entropy of the subsystem $S_{a}$,

$$
L\left(\rho_{a}\right)=2\left(1-\operatorname{Tr} \rho_{a}^{2}\right) .
$$

The Eq. (1) is understood in the following way: the right side is the entanglement between the systems $S_{a}$ and the composite system $S_{b c}$; this entanglement must be equal to the entanglement between $S_{a}$ and $S_{b}$, plus the entanglement between $S_{a}$ and $S_{c}$ and plus a genuine tripartite component $\tau$. In this way, the entanglement between $S_{a}$ and $S_{b c}$ divides itself in three well understood parts. The genuine tripartite entanglement must be interpreted as the entanglement that cannot be captured by the bipartite concurrences and that goes away when any one of the particles is lost. For instance, the state

$$
|G H Z\rangle=\frac{1}{\sqrt{2}}(|000\rangle+|111\rangle)
$$

is the typical state with only genuine tripartite entanglement. For this state, all the bipartite concurrences vanish and $\tau=1$. Also, if any one of the parties is lost, the remaining two are completely disentangled. On the other hand, the state

$$
|W\rangle=\frac{1}{\sqrt{3}}(|001\rangle+|010\rangle+|100\rangle)
$$

has only bipartite entanglement. In this case, $\tau=0$ and when one of its parties is removed, the others two remain highly entangled. In principle, there could be three distinct $\tau$ 's depending on which subsystem is chosen in the place of subsystem $S_{a}$ in Eq. (1). However, they turn out all equal for pure three qubits systems [1], showing a beautiful symmetry.

However, this symmetry ends soon as we go for more general cases. For mixed states, $\tau$ is not clearly defined 
and admits more than one generalization [17-19]. Indeed it looses the symmetry with respect to the partition chosen to write Eq. (1). In addition, also the concurrence $C_{a \mid b c}^{2}$ has to be cleared, since it is uniquely defined only for pure states. For the generalization of the concurrence, we take the usual convex roof extension $[1,11,20]$ for the linear entropy,

$$
C_{a \mid b c}^{2}=\min _{\mathcal{E}}\left\{\sum_{i} p_{i} L\left(\rho_{i}^{(a)}\right)\right\}
$$

where the minimization runs over all the ensembles $\mathcal{E}$ of pure states $\left\{p_{i}, \Phi_{i}\right\}$ such that $\rho_{a b c}=\sum_{i} p_{i} \Phi_{i}$ and $\rho_{i}^{(a)}$ is the reduced state of the subsystem $S_{a}$ when the whole system, $S_{a b c}$, is in the state $\Phi_{i}$. We notice, however, that there are other possible generalizations of the concurrence for higher dimensional systems [20, 21]. With the definition (2) for the concurrence, the following inequality is always true for any mixed state $\rho_{a b c}$ of three qubits systems [1],

$$
C_{a \mid b c}^{2}\left(\rho_{a b c}\right) \geq C^{2}\left(\rho_{a b}\right)+C^{2}\left(\rho_{a c}\right) .
$$

The inequality (3) is considered the fundamental monogamous inequality. Usually to say that a measure of entanglement is monogamous means to say that it satisfies (3). When inequality (3) is true for higher dimensional systems, others inequalities can be derived. For example, in Ref. [4], it is shown that (3) is true for the squared concurrence for systems of dimensions $2 \times 2 \times N$ and, consequently, the following inequality for $N$ qubits systems holds

$$
C_{1 \mid 23 \ldots N}^{2} \geq C_{12}^{2}+C_{13}^{2}+\cdots+C_{1 N}^{2} .
$$

These inequalities can be called second order monogamy and other interesting relations can be found [6]. However, most of the measures of entanglement turns out not monogamous, that is, usually not satisfying inequality (3) in general, with the exception of the squashed entanglement [2]. The concurrence itself fails to satisfy (3) for systems of dimensions greater or equal to $3 \times 3 \times 3$ [22]. The entanglement of formation [11] violates (3) even for three qubit systems [1]. Nevertheless, other measures of entanglement were proved monogamous in particular cases $[3,5,6]$.

One of the main points of this paper is that the inequality (3) does not exhaust the concept of monogamy. In this work, we are going to discuss a new kind of monogamy which we call multipartite. For the best of our knowledge, all the monogamous relations put in the right side of (3) an upper bound defined by a bipartite measure of entanglement. The main difference to our new monogamous inequality is that the upper bound is defined by a multipartite measure of entanglement and the left side involves the bipartite entanglement between all the possible bi-partitions. We will prove that such an inequality do exist for the concurrence in three qubit systems.
For that, first we need a generalization of the concurrence for multipartite systems. The concurrence admits many generalizations for higher dimensional and multipartite systems $[14,15,20,21]$. Between them, the generalization most interesting for our purposes here is the one by Mintert et al $[14,15]$. For a pure state $\Phi_{N}$ of an $N$ qubit system, it is defined as

$$
\mathcal{C}_{N}\left(\Phi_{N}\right)=2^{1-N / 2} \sqrt{\left(2^{N}-2\right)-\sum_{i} \operatorname{Tr} \rho_{i}}
$$

where the summation runs over all the non-trivial subsystems of the original $N$ qubit system. The interpretation of the generalized concurrence is that it captures all kinds of entanglement in a given system of $N$ particles. That is, it can be understood like the "sum" of the bipartite entanglement over all bipartition, plus the tripartite under all tripartition and so on. The main result of this work is to quantify this interpretation for three qubit systems in the following monogamous relation between the generalized concurrence, the bipartite concurrences and the genuine tripartite entanglement,

$$
\mathcal{C}_{3}^{2}\left(\Phi_{a b c}\right)=C^{2}\left(\rho_{a b}\right)+C^{2}\left(\rho_{a c}\right)+C^{2}\left(\rho_{b c}\right)+\frac{3}{2} \tau .
$$

We can understand easily the Eq. (6). The right side is a measure of all the entanglement contained in the state $\Phi_{a b c}$ while the right side is the sum of the entanglement between all the possible bi-partitions of the state plus the genuine tripartite entanglement of the state.

The proof of Eq. (6) is quite simple. First we rewrite Eq. (5) for $N=3$ as

$$
\mathcal{C}_{3}^{2}\left(\Phi_{a b c}\right)=\frac{1}{2}\left(6-\sum_{i} \operatorname{Tr} \rho_{i}^{2}\right)
$$

which is

$$
\mathcal{C}_{3}^{2}\left(\Phi_{a b c}\right)=\frac{1}{2}\left[L\left(\rho_{a}\right)+L\left(\rho_{b}\right)+L\left(\rho_{c}\right)\right],
$$

where $\rho_{i}$ is the reduced state of the subsystem $S_{i}$. Using Eq. (1) and its respective cyclic permutations we get Eq. (6). One can check that Eq. (6) shares the same interpretation of Eq. (1) with respect to the states GHZ and W. For GHZ, all the concurrences vanish and $\tau=1$, so

$$
\mathcal{C}_{3}^{2}(|G H Z\rangle)=\frac{3}{2} .
$$

For the $\mathrm{W}$ state, we have that it is $\tau$ that vanishes and

$$
\mathcal{C}_{3}^{2}(|W\rangle)=C^{2}\left(\rho_{a b}\right)+C^{2}\left(\rho_{a c}\right)+C^{2}\left(\rho_{b c}\right)=\frac{4}{3} .
$$

We can generalize the result for mixed states. In this case, the generalized concurrence is extended by the standard convex roof extension $[11,14,15,20]$. This process 
is analogous to the one which is made in Eq. (2). We define the squared generalized concurrence in Eq. (6) for a mixed state as

$$
\mathcal{C}_{N}^{2}(\rho)=\min _{\mathcal{E}}\left\{\sum_{i} p_{i} \mathcal{C}_{N}^{2}\left(\varphi_{i}\right)\right\},
$$

where the minimization goes over all the ensembles $\mathcal{E}$ of pure states $\varphi_{i}$ such that $\rho=\sum_{i} p_{i} \varphi_{i}$ [23]. Now we take a ensemble that gives the minimum in Eq. (7). For each $\varphi_{i}$ in this ensemble, the following inequality follows from Eq. (6),

$$
\mathcal{C}_{3}^{2}\left(\varphi_{i}\right) \geq C^{2}\left(\rho_{a b}^{i}\right)+C^{2}\left(\rho_{a c}^{i}\right)+C^{2}\left(\rho_{b c}^{i}\right) .
$$

Making the summation over the ensemble, we have

$$
\sum_{i} p_{i} \mathcal{C}_{3}^{2}\left(\varphi_{i}\right) \geq \sum_{i} p_{i}\left[C^{2}\left(\rho_{a b}^{i}\right)+C^{2}\left(\rho_{a c}^{i}\right)+C^{2}\left(\rho_{b c}^{i}\right)\right] .
$$

In the left side, we have $\mathcal{C}_{3}^{2}\left(\rho_{a b c}\right)$, since $\left\{p_{i}, \varphi_{i}\right\}$ is a minimizing ensemble. In the right side, from each $\varphi_{i}$ in the ensemble, we will get three density operators $\left\{\rho_{a b}^{i}, \rho_{a c}^{i}, \rho_{b c}^{i}\right\}$ which will occur with probability $p_{i}$. The right side is thus the sum of three averages over the concurrences of those density matrices. Each of these averages are made over an ensemble such that $\rho_{a b}=\sum_{i} p_{i} \rho_{a b}^{i}$ and so on. As the squared concurrence is a convex measure of entanglement, we have

$$
C^{2}\left(\rho_{a b}\right) \leq \sum_{i} p_{i} C^{2}\left(\rho_{a b}^{i}\right)
$$

and so on for the other two terms. Therefore we have the following inequality for every mixed state, $\rho_{a b c}$, of a three qubit system

$$
\mathcal{C}_{3}^{2}\left(\rho_{a b c}\right) \geq C^{2}\left(\rho_{a b}\right)+C^{2}\left(\rho_{a c}\right)+C^{2}\left(\rho_{b c}\right) .
$$

The interpretation of the inequality (8) is clear. All the entanglement contained in the state $\rho_{a b c}$ is greater or equal to the sum of the entanglements between all possible bi-partitions of the system. In addition, the inequality (8) is also an easily calculable lower bound to the mixed state multipartite concurrence. The generalized concurrence has no closed formula and also no easy calculable lower bounds for multipartite systems. Therefore, this lower bound (8) is an additional very important result per se we have gotten from the multipartite monogamy inequality (8).

One can ask whether inequality (8) can be generalized for systems of more than three qubits. The generalization of inequality (8) for an $N$ qubit system can be written as

$$
\mathcal{C}_{N}^{2} \geq \sum_{i<j} C_{i j}^{2}
$$

The simplest case is the one with four qubits. So we ask if the following inequality is true

$$
\mathcal{C}_{4}^{2} \geq C_{12}^{2}+C_{13}^{2}+C_{14}^{2}+C_{23}^{2}+C_{24}^{2}+C_{34}^{2} ?
$$

Unfortunately the answer is no and the reason seems to be simple: the generalized concurrence is not an additive measure. So, in order of showing a counter-example, let us take four qubits in a state that is a product of two maximally entangled states. In this case, the left side of inequality (10) results

$$
\mathcal{C}_{4}^{2}=\frac{1}{2^{2}}\left(2^{4}-2-\sum_{i} \operatorname{Tr} \rho_{i}^{2}\right)=\frac{1}{2^{2}}(14-7)=\frac{7}{4}
$$

while the right results

$$
C_{12}^{2}+C_{24}^{2}=2
$$

which evidently violates the inequality (10). Therefore, inequalities (9) and (10) are not true in general. This kind of counter-example is a rare event, however. Using a computer, we have generated millions of typical states according to the Haar measure and have not found any state violating the inequality (10). This fact supports our guess that it is because of the lack of the additivity property of the generalized concurrence that the inequality (10) is false. Indeed, strong connections between additivity and monogamy have already been found in Ref. [6]. In addition, the only measure known to be always monogamous is the squashed entanglement which, coincidently, is also always additive $[2,3]$.

In conclusion, we have shown a new kind of monogamy differing from the traditional ones by the use of a multipartite measure of entanglement as an upper bound to other measures. The discussion results in a beautiful relation for the generalized concurrence for pure three qubit states. The relation is extended to mixed states resulting in a monogamous inequality that we have called multipartite monogamy. Unfortunately, the result cannot be extended to more than three qubits. Nevertheless, the concept of multipartite monogamy has proved its importance in the validity of Eq. (6) and inequality (8). The fact that inequality (9), the generalization, is not always satisfied by the squared concurrence does not change the fact that multipartite monogamy is a valid and interesting concept for the study of multipartite entanglement. We expect that this work may open a new branch in the research for monogamous relations involving multipartite measures of entanglement. The study of multipartite measures is still in its beginnings and more measures are probably to come. The concept of monogamy here developed will probably be important for the judgment of good measures of multipartite entanglement. The Eq. (6) and inequality (8) may be the firsts of a series of new monogamous relations that multipartite measures should satisfy. In this sense, we believe that this work goes beyond showing a new monogamous relations, it opens a new branch of research for monogamy and multipartite entanglement. 
[1] V. Coffman, J. Kundu, and W. K. Wootters, Physical Review A 61, 052306 (2000).

[2] M. Christandl and A. Winter, Journal of Mathematical Physics 45, 829 (2004).

[3] M. Koashi and A. Winter, Physical Review A 69, 022309 (2004).

[4] T. J. Osborne and F. Verstraete, Physical Review Letters 96, 220503 (2006).

[5] G. Adesso and F. Illuminati, Physical Review Letters 99, 150501 (2007).

[6] M. F. Cornelio and M. C. de Oliveira, Physical Review A 81, 032332 (2010).

[7] M. Pawłowski, Physical Review A 82, 032313 (2010).

[8] M. Pawłowski and Č. Brukner, Physical Review Letters 102, 030403 (2009).

[9] A. Streltsov, G. Adesso, M. Piani, and D. Bruß, Physical Review Letters 109, 050503 (2012).

[10] H. Ollivier and W. H. Zurek, Physical Review Letters 88, 017901 (2001).

[11] C. H. Bennett, D. P. DiVincenzo, J. A. Smolin, and W. K. Wootters, Physical Review A 54, 3824 (1996).

[12] M. F. Cornelio, M. C. de Oliveira, and F. F. Fanchini, Phys. Rev. Lett. 107, 020502 (2011).

[13] F. F. Fanchini, M. F. Cornelio, M. C. de Oliveira, and
A. O. Caldeira, Physical Review A 84, 012313 (2011), arXiv:1006.2460.

[14] F. Mintert, M. Kuś, and A. Buchleitner, Physical Review Letters 95, 260502 (2005).

[15] F. Mintert, A. R. R. Carvalho, M. Kuś, and A. Buchleitner, Physics Reports 415, 207 (2005).

[16] W. K. Wootters, Physical Review Letters 80, 2245 (1998).

[17] C. Eltschka, A. Osterloh, J. Siewert, and A. Uhlmann, New Journal of Physics 10, 043014 (2008).

[18] E. Jung, M.-R. Hwang, D. K. Park, and J.-W. Son, Physical Review A 79, 024306 (2009).

[19] K. Cao, Z.-w. Zhou, G.-c. Guo, and L. He, Physical Review A 81, 034302 (2010).

[20] A. Uhlmann, Physical Review A 62, 032307 (2000).

[21] P. Rungta, V. Bužek, C. M. Caves, M. Hillery, and G. J. Milburn, Physical Review A 64, 042315 (2001).

[22] Y.-C. Ou, Physical Review A 75, 034305 (2007).

[23] This definition differs from the one originally given by Mintert et al [14] for the generalized concurrence of mixed states, namely $\mathcal{C}_{N}(\rho)=\min _{\mathcal{E}}\left\{\sum_{i} p_{i} \mathcal{C}_{N}\left(\varphi_{i}\right)\right\}$. This difference resembles the one between the Iconcurrence (without the squared) and the tangle (with the squared) for the concurrence of bipartite systems of dimensions higher than two. In principle, none can be claimed better than the other, but the notion of the tangle results in the CKW monogamy for mixed states and, here, in the multipartite monogamy. 